\title{
The outer filament of Centaurus A as seen by MUSE
}

\author{
F. Santoro ${ }^{1,2}$, J. B. R. Oonk ${ }^{1,3}$, R. Morganti ${ }^{1,2}$, T. A. Oosterloo ${ }^{1,2}$, and G. Tremblay ${ }^{4}$ \\ 1 ASTRON, Netherlands Institute for Radio Astronomy, PO 2, 7990 AA Dwingeloo, The Netherlands \\ e-mail: santoro@astro.rug.nl \\ 2 Kapteyn Astronomical Institute, University of Groningen, PO 800, 9700 AV Groningen, The Netherlands \\ 3 Leiden Observatory, Leiden University, PO Box 9513, 2300 RA Leiden, The Netherlands \\ 4 Yale University, Department of Physics, 217 Prospect St., New Haven, CT 06511, USA
}

Received 12 December 2014 / Accepted 23 January 2015

\section{ABSTRACT}

\begin{abstract}
Context. Radio-loud active galactic nuclei (AGN) are known to inject kinetic energy into the surrounding interstellar medium (ISM) of their host galaxy via plasma jets. Understanding the impact that these flows can have on the host galaxy helps to characterize a crucial phase in their evolution. Because of its proximity, Centaurus A is an excellent laboratory in which the physics of the coupling of jet mechanical energy to the surrounding medium may be investigated. About $15 \mathrm{kpc}$ northeast of this galaxy, a particularly complex region is found: the so-called outer filament, where jet-cloud interactions have been proposed to occur.

Aims. We investigate signatures of a jet-ISM interaction using optical integral-field observations of this region, expanding on previous results that were obtained on a more limited area.

Methods. Using the Multi Unit Spectroscopic Explorer (MUSE) on the VLT during the science verification period, we observed two regions that together cover a significant fraction of the brighter emitting gas across the outer filament. Emission from a number of lines, among which $\mathrm{H} \beta \lambda 4861 \AA$, [O III] $\lambda \lambda 4959,5007 \AA, \mathrm{H} \alpha \lambda 6563 \AA$, and [N II] $\lambda \lambda 6548,6584 \AA$, is detected in both regions.

Results. The ionized gas shows a complex morphology with compact blobs, arc-like structures, and diffuse emission. Based on the kinematics, we identified three main components of ionized gas. Interestingly, their morphology is very different. The more collimated component is oriented along the direction of the radio jet. The other two components exhibit a diffuse morphology together with arclike structures, which are also oriented along the radio jet direction. Furthermore, the ionization level of the gas, as traced by the $[\mathrm{O}$ III $] \lambda 5007 / \mathrm{H} \beta$ ratio, is found to decrease from the more collimated component to the more diffuse components.

Conclusions. The morphology and velocities of the more collimated component confirm the results of our previous study, which was limited to a smaller area, implying that both the outer filament and the nearby H I cloud are probably partially shaped by the lateral expansion of the jet. The arc-like structures embedded within the two remaining components are the clearest evidence of a smooth jet-ISM interaction along the jet direction. We thus find signs of a jet-ISM interaction across all identified gas components. This suggests that, although poorly collimated, the large-scale radio jet is still active and affects the surrounding gas. This result indicates that the effect on the ISM of even low-power radio jets should be considered when studying the influence AGN can have on their host galaxy.
\end{abstract}

Key words. galaxies: active - ISM: jets and outflows - galaxies: individual: Centaurus A

\section{Introduction}

Active galactic nuclei (AGN) can influence the evolution of their host galaxy by injecting energy into the surrounding interstellar medium (ISM; see Heckman \& Best 2014, for a review). The mechanical energy provided by a radio-loud AGN is mainly in the form of twin jets launched into the ISM, which can represent a key ingredient in the overall evolution of an active galaxy. Indeed, the interaction between radio jets and ISM has been found to be relevant at large and small scales both by observations (see McNamara \& Nulsen 2012; Morganti et al. 2013, and references therein) and numerical simulations (e.g., Gaibler et al. 2012; Wagner et al. 2012).

How the interaction between the jet and the ISM proceeds, how it depends on the kinetic power of the jet, for instance, and whether this process is still strong in the more common lowpower radio galaxies, are some of the open questions. Several gas outflows are known that are driven by low-power radio jets, including cases of massive neutral and molecular outflows (e.g., Alatalo et al. 2011; Combes et al. 2013). This indicates the possibility that even jets with low kinetic power can drive outflows, suggesting that the jet-ISM interaction could be a relatively common phenomenon associated with the active phase of a galaxy (see also Harrison et al. 2014, for a discussion).

Detailed observations are a crucial starting point for understanding the physics of jet-ISM interactions. Furthermore, they can place important constraints on the main parameters of the models used to simulate these interactions. Signatures of jet-ISM interactions have also been found in the nearby AGN Centaurus A (Cen A, see Rejkuba et al. 2002; Oosterloo \& Morganti 2005; Hamer et al. 2015; Santoro et al. 2015). This led us to choose this galaxy as a target for a detailed study of the jet-ISM interaction phenomenon. Cen $\mathrm{A}$ is a relatively lowpower radio galaxy, $\left(P_{2.7 \mathrm{GHz}}=1.8 \times 10^{24} \mathrm{WHz}^{-1}\right.$; Morganti et al. 1999) as well as the nearest observed $\operatorname{AGN}(d=3.8 \mathrm{Mpc}$; Harris et al. 2010, for which 1 arcmin $=1 \mathrm{kpc}$ ), with a systemic velocity $v_{\text {sys }} \sim 540 \mathrm{~km} \mathrm{~s}^{-1}$. Filaments of highly ionized gas have been found at different locations along the jet direction (Blanco et al. 1975; Graham \& Price 1981; Morganti et al. 1991). Past studies mainly focused on the so-called inner and outer filaments situated at a distance of about $8.5 \mathrm{kpc}$ and $15 \mathrm{kpc}$ from the galaxy nucleus. In both cases, the radio plasma jet is thought to affect the characteristics of the gas. Indeed, these filaments are close to the inner radio lobe and to the large-scale jet (see e.g. discussion in Morganti et al. 1999). 
The outer filament region presents a particularly intriguing situation, where a complex interplay between radio plasma and neutral and ionized gas is ongoing. Interestingly, only a low surface brightness and poorly collimated jet-like structure has been observed in this region (Morganti et al. 1999). Whether this structure corresponds to a still active jet or to a relic is still a matter of discussion (e.g., Saxton et al. 2001; Hardcastle et al. 2009). Indirect evidence of interaction - with associated star formation - at this location has been found by a number of studies (Mould et al. 2000; Rejkuba et al. 2002; Oosterloo \& Morganti 2005). Oosterloo \& Morganti (2005) studied the kinematics of the H I cloud, located - in projection - close to the outer filament and found that gas with anomalous velocities is a possible interaction signature.

Santoro et al. (2015) carried out an integral-field spectroscopic study of the ionized gas using the Visible MultiObject Spectrograph (VIMOS) on two small regions of the outer filament. They found two kinematical components whose velocities match those of the two components found in the nearby H I cloud. These data support the idea that ionized and neutral gas are likely part of a single dynamical structure that is partly affected by the interaction with a poorly collimated, but active jet. If this is the case, the kinematical signature of the jet-ISM interaction traced by Santoro et al. (2015) should be seen over a larger area of the outer filament. With the goal of extending our study over a larger region, we have exploited the new capabilities provided by the Multi Unit Spectroscopic Explorer (MUSE, Bacon et al. 2010) integral field spectrograph that was recently installed at the ESO/Very Large Telescope (VLT). In this letter we show the first results on the kinematics of the gas, while a detailed analysis of the ionization and line ratios will be presented in a forthcoming paper.

\section{Data reduction and analysis}

We observed two fields in the outer filament of Cen A using MUSE at the VLT. Observations were carried out on June 25, 2014 during the science verification program with an exposure time of $3 \times 16.6 \mathrm{~min}$ for each field. Our two MUSE pointings cover the brighter region of the outer filament, which partially overlaps the our previous VIMOS observations (Santoro et al. 2015). Compared to that study, we increased the observed area from $\sim 0.5$ to $2 \operatorname{arcmin}^{2}$ and now cover a large part of the outer filament. Furthermore, we extend the observed wavelength range beyond the $6200 \AA$ VIMOS limit to $9300 \AA$ with MUSE. This enables us to include the important diagnostic lines from the red part of the optical spectrum.

The observations were made in Wide Field Mode with a field of view (FoV) covering $1 \times 1 \mathrm{arcmin}^{2}$ and a pixel size of $0.2 \times 0.2 \operatorname{arcsec}^{2}$. The spatial resolution is limited by the seeing, which was $\sim 1$ arcsec. The spectral range is about 4700-9300 and the spectral resolution is $\sim 2.3 \AA$, corresponding to a velocity resolution ranging from $75 \mathrm{~km} \mathrm{~s}^{-1}$ at the longest wavelengths to $150 \mathrm{~km} \mathrm{~s}^{-1}$ at the shortest one. For flux calibration purposes we used the standard calibration procedure. The data reduction was carried out using the ESO pipeline (Version 0.18.2) with default parameters (Weilbacher et al. 2014). The sky subtraction process left residuals at the location of bright sky lines, but these do not compromise the quality of the science discussed here. Emission lines from ionized gas are clearly detected over the whole wavelength range and across both fields and include $\mathrm{H} \beta \lambda 4861 \AA$, [O III] $] \lambda 44959,5007 \AA, H \alpha \lambda 6563 \AA,[N$ II $] \lambda \lambda 6548,6584 \AA$,
[S II] $] \lambda 6717,6731 \AA$, and [O I] $\lambda \lambda 6300,6363 \AA$. All the lines show the same spatial and velocity distribution.

\section{Results}

The integrated emission of $\mathrm{H} \alpha$ given in Fig. 1 (left panel) shows an intriguing variety of gas structures spread over the entire observed region. The gas distribution we observe across our FoV is consistent with the previous optical studies of the outer filament (Morganti et al. 1991; Graham 1998; Rejkuba et al. 2001; Santoro et al. 2015), but we obtain for the first time detailed kinematical information over a large area of the filament. The gas is distributed in structures that range from compact and bright to faint, extended, and diffuse. Particularly interesting are the welldefined arc-like features in the diffuse emission. The MUSE data allow us to distinguish three kinematical components, which are represented in Fig. 1 (right panel). This figure shows an RGB image of the $\mathrm{H} \alpha$ emission colour-coded based on the heliocentric velocity of the line emission. Here we have separated the components by summing the line emission in three heliocentric velocity ranges: -108-177 $\mathrm{km} \mathrm{s}^{-1}$ (blue), 177-405 $\mathrm{km} \mathrm{s}^{-1}$ (green), $405-745 \mathrm{~km} \mathrm{~s}^{-1}$ (red). All these structures are blueshifted relative to the systemic velocity of Cen A. Two components (shown in green and red) correspond - extending over a larger area to those reported by Santoro et al. (2015). However, our new data reveal a third, lower velocity component (shown in blue). Because of the limited FoV, it was not detected in the VIMOS study. Hints of this component were previously seen in the long-slit study of Graham (1998). In the following we describe the main kinematical and morphological features of these three components.

Linear component. Over the velocity range 405-745 $\mathrm{km} \mathrm{s}^{-1}$, at an intensity-weighted mean heliocentric velocity of $\sim 450 \mathrm{~km} \mathrm{~s}^{-1}$, we find a well-defined linear, knotty filament. This component is shown in red in the right panel of Fig. 1 and has a southwest-northeast orientation, similar to that of the large-scale jet. The average value of the $[\mathrm{O} \mathrm{III}] \lambda 5007 / \mathrm{H} \beta$ ratio is $\sim 6.4_{-0.3}^{+0.3}$, while for $[\mathrm{N}$ II $] \lambda 6584 / \mathrm{H} \alpha$ it is $\sim 0.56_{-0.07}^{+0.09}$. An interesting kinematical feature can be seen in one specific location within this component. In Fig. 2 we show the positionvelocity diagram of this feature extracted along the green line drawn in the left panel of Fig. 1. Over a well-defined spatial extent (of $\sim 70 \mathrm{pc}$ ), the heliocentric velocities of the gas are 300-350 $\mathrm{km} \mathrm{s}^{-1}$ higher than those of the surrounding gas (at $v_{\text {hel }} \sim 450 \mathrm{~km} \mathrm{~s}^{-1}$ ). The position-velocity diagram shows that the gas gradually reaches higher velocities, creating a continuous velocity structure that gives the impression of a small bubble being accelerated. At this location, the brightness of the filament is markedly lower than in the surrounding regions.

Diffuse component. This component is shown in green in the right panel of Fig. 1 and has an intensity-weighted mean heliocentric velocity of $\sim 260 \mathrm{~km} \mathrm{~s}^{-1}$. Morphologically, this component appears to be very different from the linear component described above. While some structure on small scales is present, most of the emission comes from extended, diffuse gas that gives the impression of gas filaments being blown to the northeast. Reinforcing this idea, several arc-like clumps with a bow-shock shape can be clearly identified in the southern field. They vary in size, but all have a semicircular shape and the same orientation. This diffuse component has a velocity similar, although not identical, to that of the quiescent neutral gas of the nearby $\mathrm{H}$ I cloud, and we suggest that it originates from the H I structure. For the diffuse component the average [O III $] \lambda 5007 / \mathrm{H} \beta$ ratio is $\sim 3_{-0.17}^{+0.19}$ while for the $[\mathrm{N}$ II $] \lambda 6584 / \mathrm{H} \alpha$ it is $\sim 0.65_{-0.07}^{+0.08}$. 


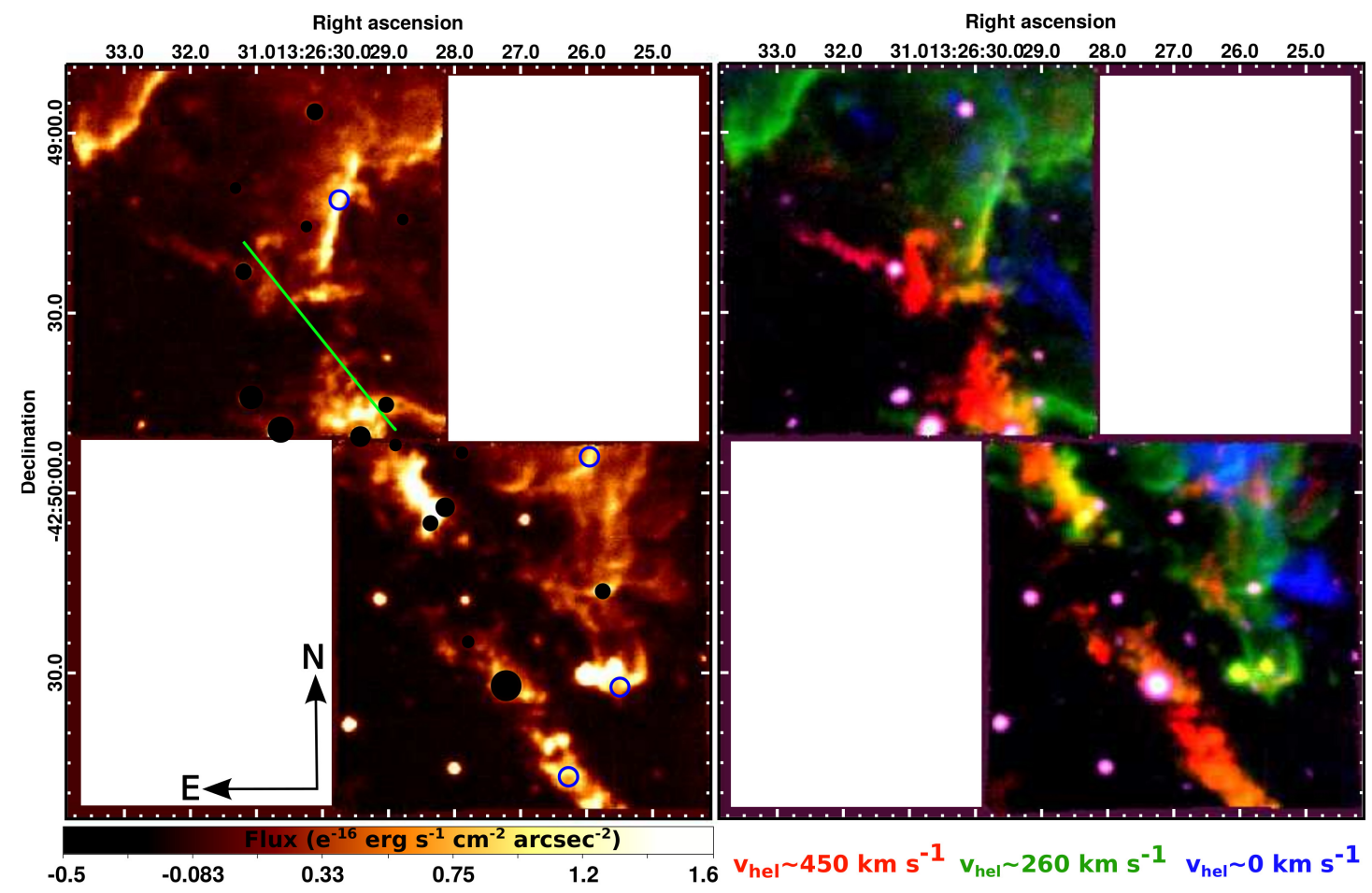

Fig. 1. Left panel: total $\mathrm{H} \alpha$ line flux map. Blue circles mark the regions from which the spectra shown in Fig. 3 are extracted (from top to bottom: diffuse component, low-velocity component, arc-like clumps, linear component). Black-filled circles mark the stellar light that is superimposed on the ionized gas emission. The location of the position-velocity plot of Fig. 2 is indicated with a green line. Right panel: RGB image of the H $\alpha$ emission showing the linear (red), the diffuse (green) and the low-velocity (blue) components. At the bottom we indicate the intensity weighted mean heliocentric velocity of each component; note that Cen A $v_{\mathrm{sys}} \sim 540 \mathrm{~km} \mathrm{~s}^{-1}$. The regions where the overlap between the linear and the diffuse components is more evident are plotted in yellow.

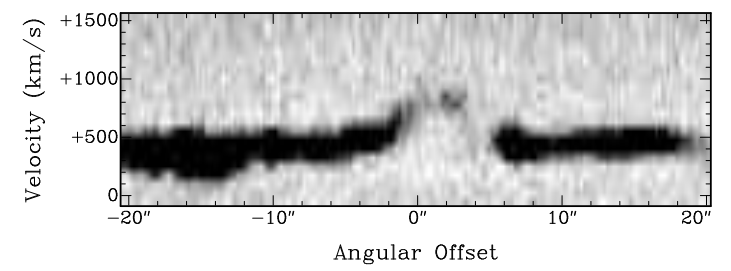

Fig. 2. Position-velocity plot of the [O III] $\lambda 5007 \AA$ A emission taken along the line indicated in Fig. 1 (left panel). The velocities are heliocentric.

Similar average values are also found in the arc-like clumps: $[\mathrm{O} \mathrm{III]}] \lambda 5007 / \mathrm{H} \beta \sim 4_{-0.22}^{+0.25}$ and $[\mathrm{N} \mathrm{II}] \lambda 6584 / \mathrm{H} \alpha \sim 0.64_{-0.07}^{+0.07}$. The morphology and the velocities of the linear and the diffuse components match - in the overlapping region - those of the highand low-velocity components observed by Santoro et al. (2015).

Low-velocity component. At an intensity-weighted mean heliocentric velocity of $\sim 0 \mathrm{~km} \mathrm{~s}^{-1}$, we identify a third component. It is shown in blue in the right panel of Fig. 1 and has fainter emission than the other two components. Morphologically, this component is very similar to the diffuse component described above. It is composed of diffuse emission and, in the lower field, shows a single arc-like structure whose morphology is similar to the arc-like clumps found in the diffuse component. The average value of the $[\mathrm{O}$ III $] \lambda 5007 / \mathrm{H} \beta$ ratio is $\sim 1.6_{-0.64}^{+0.72}$, while for the [N II] $] 26584 / \mathrm{H} \alpha$ ratio it is $\sim 0.81_{-0.11}^{+0.14}$.

The average line ratios reported above for the different components are the average of many individual regions extracted for each component. In Fig. 3 we show a representative spectrum from one of these regions for each component. We mark the regions from which these spectra are extracted in the left panel of Fig. 1. The average line ratios show that the ionization state of the gas is different in the three different components. We assume $[\mathrm{N} \mathrm{II}] \lambda 6584 / \mathrm{H} \alpha \approx 0.6$ and $[\mathrm{O}$ III $] \lambda 5007 / \mathrm{H} \beta \approx 2.0$ as typical dividing values for H II region/AGN and AGN/lowionization nuclear emission-line region (LINER), respectively (see Kewley et al. 2006). From comparing the extracted line ratios with these values, we confirm earlier results that ruled out that the ionization of the gas is due to stellar light.

\section{Discussion and conclusions}

The MUSE observations reveal the velocity structure of the ionized gas over a large region of the outer filament of Cen A at high spatial resolution. The morphology and velocities of the ionized gas in the overlapping region agree with the results of Santoro et al. (2015). However, the two components that match the kinematics of the components in the nearby H I cloud are now found to cover a much larger area of the filament. Thus, our data support the results and interpretation from the VIMOS data that the gas is affected by the interaction with the radio jet, and in particular, that this is the case for a much larger area and, by implication, probably for the entire outer filament. The MUSE data also show that the situation is even more complex. Interestingly, the different kinematical components now also appear to be connected with different morphologies (linear, arc-like, and diffuse). Furthermore, we identified a new low-velocity component.

For the outer filament we propose that the diffuse and the low-velocity components are directly affected by the passage of a slow-moving jet. A smooth interaction of this kind would, indeed, explain the arc-like clumps embedded within them. They show a striking similarity with the structures described by Bicknell (1991). These simulations show that arc-shaped 


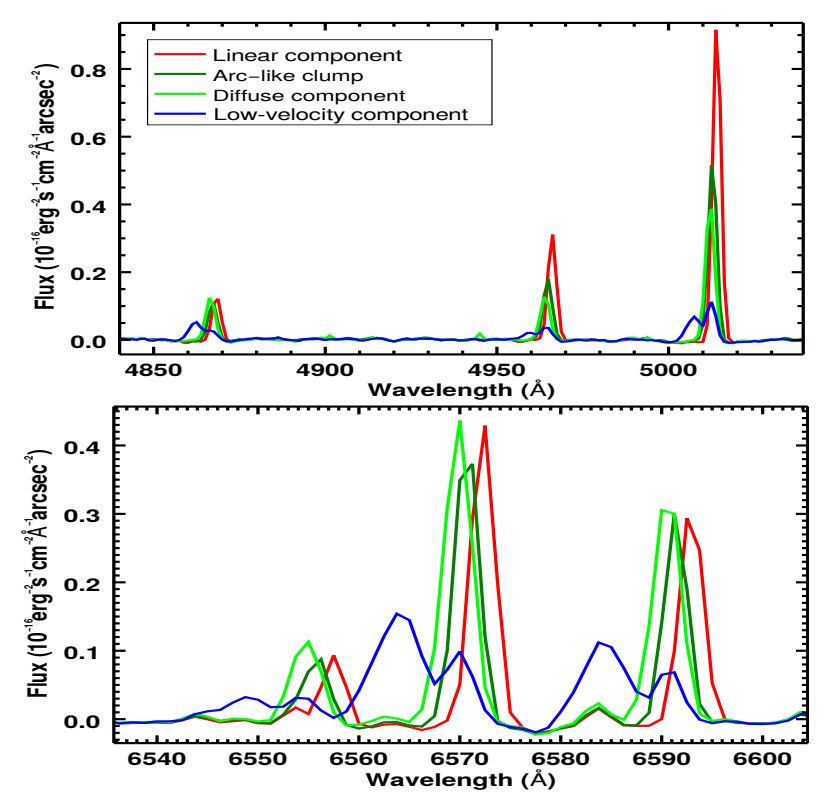

Fig. 3. Spectra extracted from the regions indicated in Fig. 1 (left panel). The spectra are given for the $\mathrm{H} \beta,[\mathrm{O}$ III] $] \lambda 44959,5007 \AA$ (upper panel) and the $\mathrm{H} \alpha,[\mathrm{N}$ II $] \lambda \lambda 6548,6584 \AA$ (lower panel) lines. Because it overlaps the diffuse component, the spectrum extracted for the low-velocity component shows double-peaked emission lines. The peak associated with the latter component is at lower wavelengths.

structures can form as a result of the encounter between a transonic stream of non-thermal plasma and denser clouds of thermal gas. The orientation of the large-scale jet and the arc-like structures agrees with the results from such simulations. In addition, the nature of the large-scale jet of Cen $\mathrm{A}$ is compatible with a low-Mach number jet as used in the simulation. Consistent with our previous study, we find that the linear component is characterized by velocities similar to the H I anomalous velocities, which supports the scenario described in Santoro et al. (2015). This component represents the part of the H I cloud that through its rotation about the galaxy has entered the zone of influence of the large-scale radio jet. The gas in this region is probably affected by the lateral expansion of the jet cocoon. The well-defined elongation of the linear component along the same direction as the jet is possibly the result of this lateral expansion. These characteristics, and the compression that may result, could also explain the interesting kinematical structure illustrated in Fig. 2. These structures are reminiscent of a bursting over-pressured bubble. It is unclear whether the newly found low-velocity component is a possible counterpart of this linear component (i.e., also affected by a lateral expansion) but on the other side of the jet and, as a consequence, characterized by low velocities. The very different morphologies of these two components could be due to intrinsic differences in the density or distribution of the gas within the two regions.

Although the line ratios found for all components, when plotted in a BPT diagram (Baldwin et al. 1981), are typical of AGN/LINER, we note a trend to be present, with the linear component showing higher excitation levels. This may reflect a change in the ionized gas density or in the number of ionizing photons across the region we are observing. Apart from the low-velocity component, for which the full-width-at-halfmaximum (FWHM) of the $\mathrm{H} \alpha$ line is $\sim 4.5 \AA$ (corresponding to $\sim 205 \mathrm{~km} \mathrm{~s}^{-1}$ ), the narrow velocity width of the $\mathrm{H} \alpha$ lines of the other components $(F W H M \sim 3 \AA$ corresponding to $\sim 137 \mathrm{~km} \mathrm{~s}^{-1}$ ) leads us to argue (as already discussed in Santoro et al. 2015) that the jet does not drive a strong shock across the whole observed region. A more detailed analysis of the kinematics and ionization will be presented in a forthcoming paper.

It is worth noting that we find similarities with the results recently obtained (also using MUSE) for the inner filament of Cen A (Hamer et al. 2015). Indeed, both the inner and the outer filaments contain a well-defined linear knotty plus a more diffuse structure that show different kinematics and line ratios. It is thus possible that similar mechanisms are responsible for the kinematics and the ionization state of the ionized gas within both the inner and the outer filament.

In summary, the general implications of our study are that the kinematics of the gas supports the idea that the large-scale jet is still an active structure, but is characterized, as expected from its morphology, by relatively low (transonic) velocities. Despite being a low-power jet, the effects of its lateral and head-on interaction with the surrounding ISM are clearly visible, and this is the first time that we can trace the gas and its kinematics at the location of a jet-cloud interaction on such small scales. This shows that the jet-ISM interaction has to be considered when studying the effect of the feedback on a galaxy that hosts a lowpower radio source.

Acknowledgements. G.T. would like to thank Bernd Husemann for his help with the MUSE pipeline. The research leading to these results has received funding from the European Research Council under the European Union's Seventh Framework Programme (FP/2007-2013)/ERC Advanced Grant RADIOLIFE320745. Based on observations made with ESO Telescopes at the La Silla Paranal Observatory under programme 60.A-9341A.

\section{References}

Alatalo, K., Blitz, L., Young, L. M., et al. 2011, ApJ, 735, 88

Bacon, R., Accardo, M., Adjali, L., et al. 2010, SPIE Conf. Ser., 7735, 8

Baldwin, J. A., Phillips, M. M., \& Terlevich, R. 1981, PASP, 93, 5

Bicknell, G. 1991, PASA, 9, 93

Blanco, V., Graham, J. A., Lasker, B. M., \& Osmer, P. S. 1975, ApJ, 198, L63

Combes, F., García-Burillo, S., Casasola, V., et al. 2013, A\&A, 558, A124

Gaibler, V., Khochfar, S., Krause, M., \& Silk, J. 2012, MNRAS, 425, 438

Graham, J. A. 1998, ApJ, 502, 245

Graham, J. A., \& Price, R. M. 1981, ApJ, 247, 813

Hamer, S., Salomé, P., Combes, F., \& Salomé, Q. 2015, A\&A, 575, L3

Hardcastle, M., Cheung, C. C., Feain, I., \& Stawarz, Ł. 2009, MNRAS, 393 1041

Harris, G. L. H., Rejkuba, M., \& Harris, W. E. 2010, PASA, 27, 457

Harrison, C., Alexander, D., Mullaney, J., \& Swinbank, A. 2014, MNRAS, 441, 3306

Heckman, T. M., \& Best, P. N. 2014, ARA\&A, 52, 589

Kewley, L., Groves, B., Kauffmann, G., \& Heckman, T. 2006, MNRAS, 372, 961

McNamara, B. R., \& Nulsen, P. E. J. 2012, New J. Phys., 14, 055023

Morganti, R., Robinson, A., Fosbury, R. A. E., et al. 1991, MNRAS, 249, 91

Morganti, R., Killeen, N., Ekers, R., \& Oosterloo, T. 1999, MNRAS, 307, 750

Morganti, R., Fogasy, J., Paragi, Z., Oosterloo, T., \& Orienti, M. 2013, Science, 341,1082

Mould, J. R., Ridgewell, A., Gallagher, III, J. S., et al. 2000, ApJ, 536, 266 Oosterloo, T. A., \& Morganti, R. 2005, A\&A, 429, 469

Rejkuba, M., Minniti, D., Silva, D. R., \& Bedding, T. R. 2001, A\&A, 379, 781 Rejkuba, M., Minniti, D., Courbin, F., \& Silva, D. R. 2002, ApJ, 564, 688

Santoro, F., Oonk, J. B. R., Morganti, R., \& Oosterloo, T. 2015, A\&A, 574, A89 Saxton, C. J., Sutherland, R. S., \& Bicknell, G. V. 2001, ApJ, 563, 103 Wagner, A. Y., Bicknell, G. V., \& Umemura, M. 2012, ApJ, 757, 136

Weilbacher, P. M., Streicher, O., Urrutia, T., et al. 2014, in Astronomical Data Analysis Software and Systems XXIII, eds. N. Manset, \& P. Forshay, ASP Conf. Ser., 485, 451 